\title{
MÉTODOS PARA EXTRAÇÃO DE ÓLEO DAS SEMENTES DA Theobroma grandiflorum COMO OPORTUNIDADE A AGROINDUSTRIA FAMILIAR
}

\section{METHODS FOR EXTRACTION OF Theobroma grandiflorum OIL FROM SEEDS AS AN OPPORTUNITY TO FAMILY AGROINDUSTRY}

\author{
Julia De Lourdes da Silva ${ }^{1}$ \\ Maria Fernanda Berlingieri Durigan ${ }^{2}$ \\ Gabriella Ferreira Carvalho ${ }^{3}$
}

RESUMO: No Brasil, o cupuaçu possui uma cadeia produtiva muito importante, principalmente para pequenos produtores rurais. É popular no país todo, sendo mais produzido em sua região de origem, na região Amazônica, e no nordeste brasileiro. É uma fruta com diversas aptidões. As mais populares são o uso das cascas e das sementes para compostagem e a polpa vendida principalmente como polpa congelada para sucos, cremes e sorvetes. A manteiga, feita a partir do óleo das sementes de cupuaçu, tem se tornado cada vez mais popular, sendo utilizado em pequenas e grandes indústrias de cosméticos devido a presença de ácidos graxos e benefícios interessantes para saúde da pele e cabelo, ótimo emoliente e alto índice de saponificação. Porém a extração do óleo ou manteiga não é popular ou incentivada entre os produtores da fruta ou da polpa, que geralmente descartam as sementes, em grande quantidade, desperdiçando oportunidades de renda. Com o intuito de organizar informações sobre o assunto, foi elaborada uma revisão de literatura, que teve como objetivo buscar artigos que exemplificassem e descrevessem, por meio de informações básicas, as principais informações sobre o óleo de cupuaçu e os métodos de extração mais utilizados, principalmente aqueles acessíveis a agroindústria familiar. Para a coleta de dados foram utilizadas as bases de dados das

\footnotetext{
${ }^{1}$ Química, Mestranda em Agroecologia pela Universidade Estadual de Roraima. E-mail: julia.lourdes.s@hotmail.com.

${ }^{2}$ Pesquisadora na Empresa Brasileira de Pesquisa Agropecuária (EMBRAPA-RR). E-mail: maria.durigan@embrapa.br.

${ }^{3}$ Acadêmica do curso de Agronomia na faculdade FARES. E-mail: gabicarvalho.rr7@ gmail.com
} 
plataformas Scielo, Google Acadêmico e a Base de Dados da Embrapa (Empresa Brasileira de Pesquisa Agropecuária). Foram encontrados métodos de extração por meio de prensas ou solventes e informações que podem contribuir para a valorização e popularização do óleo de cupuaçu, assim como incentivar a extração agroindustrial do mesmo.

Palavras-chave: Extrativismo, produto florestal não madeireiro (PFNM), manteiga, cupulate.

ABSTRACT: In Brazil, cupuaçu has a very important productive chain, mainly for small farmers. It is popular in the whole country, being produced in its region of origin, in the Amazon region, and in the Brazilian northeast. It is a fruit with several aptitudes. The most popular are the use of peels and seeds for composting and the pulp sold mainly as frozen pulp for juices, creams and ice creams. The butter, made from cupuaçu seed oil, has become increasingly popular, being used in small and large cosmetics industries due to the presence of fatty acids and interesting benefits to skin and hair health, great emollient and high health saponification index. However, the extraction of oil or butter is not popular or encouraged among fruit or pulp producers, who generally discard seeds in large quantities, wasting income opportunities. In order to organize information on the subject, an review of available literature was elaborated, with the objective of finding articles that would exemplify and describe, through basic information, the main information about cupuaçu oil and the most extracted extraction methods used, especially those accessible to family agroindustry. For data collection, the databases of the Scielo, Google Academic and Embrapa Database (Brazilian Company for Agricultural Research) were used. Extraction methods were found by means of presses or solvents and information that can contribute to the valorization and popularization of cupuaçu oil, as well as to encourage the agroindustrial extraction of the same.

Keywords: Extractivism, non-timber forest product, butter, cupulate. 


\title{
INTRODUÇÃO
}

Na Amazônia há uma diversidade abundante de espécies com grande potencial para produção e comercialização. Esses produtos são uma das maiores fontes de renda para pequenos e grandes agricultores, oriundos das atividades agrícolas e industriais, gerando empregos e promovendo exportação (Said, 2011).

A arvore de cupuaçu, o cupuaçuzeiro, pode alcançar a altura de até $15 \mathrm{~m}$, com diâmetro que pode atingir de 6 a $8 \mathrm{~m}$. (Santos, 2016). Quanto ao fruto do cupuaçu é uma baga, que possui uma casca dura de forma elíptica e pode pesar em média 1,500 kg, e suas semente representam uma fração significativa do fruto.

\begin{abstract}
"As sementes do cupuaçu apresentam-se em número de 35 a 50 por fruto e correspondem a 15 a $21,1 \%$ do peso deste, estando distribuída em torno de um eixo central, a placenta, disposta de forma longitudinal em relação ao comprimento do fruto. As sementes encontram-se firmemente protegidas pela polpa, que é abundante, de fibras longas, coloração amarelada ou esbranquiçada, sabor ácido e odor forte. Possuem forma circular achatada com dimensões médias de 2,5 a 2,6 cm de diâmetro por 2,0 a 2,3 cm de largura e 0,9 a $1,1 \mathrm{~cm}$ de espessura (Said, 2011, p. 42)."
\end{abstract}

Os frutos são comercializados e vendidos por todo o país, bem como exportados com boa aceitação. Por possuir metabolismo acelerado que catalisa seu processo de amadurecimento, há dificuldades no que se diz respeito ao seu armazenamento in natura, com isso a procura pela produção e comercialização de polpas congeladas dessa fruta é o meio mais popular de comercialização, facilitando o transporte e a conservação dos produtos (Dal Ri, 2006).

A produção de cupuaçu provém, basicamente, de plantios comerciais, estimados em mais de 20.000 ha, distribuídos nos estados do Pará, Amazonas, Rondônia e Acre, principalmente. No Amazonas quase todos os municípios possuem pequenas áreas produtivas com o cupuaçuzeiro, tornando-se, portanto, um dos produtos mais expressivos do estado (Alves et al., 2014).

Em Roraima o cupuaçu é de extrema importância para os pequenos produtores rurais e é normalmente cultivado em Sistemas Agroflorestais (SAFs). O aumento do 
investimento local nesta espécie está relacionado a boa produção, considerada alta, em torno de $1.200 \mathrm{~kg} \mathrm{ha}^{-1}$, principalmente no sul do estado (Lima et al, 2013).

Há grande diversidade de subprodutos obtidos da polpa à semente, muito utilizados industrialmente por seu odor e sabor agradável e característico. A polpa é popularmente utilizada para o preparo de sucos, mouses e bolos (Lannes; Medeiros; Amaral, 2002). A semente é geralmente descartada, sendo utilizada em alguns casos para compostagem ou para produção de novas plantas.

De acordo com a Comissão Nacional de Normas e Padrões para Alimentos do Ministério da Saúde do Brasil se entende por óleos e gorduras comestíveis, na Resolução $\mathrm{N}^{\circ}$. 22/77, os produtos que contém glicerídeos de ácidos gordurosos de origem vegetal ou animal, onde pode existir em pouca quantidade. Para melhor diferenciar gorduras de óleos se observa o estado físico do produto, onde as gorduras assumem um estado sólido e acabam sendo chamados também de manteiga e o óleo permanece no estado líquido (Azevedo, 2001).

Dentro desse contexto se encontra o óleo do cupuaçu, produto que tem chamado atenção dos produtores por suas possibilidades. A planta é da mesma família do cacau e possui grande percentual de gordura disponível nas sementes, geralmente são tratadas como descarte, ou acabam sendo utilizadas para ração ou adubo que, apesar de ser um meio de reutilização do produto, ainda é uma forma que subutiliza o potencial nutritivo dessas sementes (Medeiros et al., 2006).

A semente do cupuaçu é geralmente descartada ou utilizada para a produção de novas plantas. Com conteúdo considerável de óleo, é comparada a gordura do cacau que, após fermentadas e secas, pode-se obter o óleo de cupuaçu. Devido a esta comparação com o cacau, produtos oriundos dessa semente são chamados de cupulate. Mesmo com o tratamento de fermentação e secagem, estudos vem mostrando um alto teor de nutrientes provenientes das sementes. Com um teor de 9 a $12 \%$ de proteínas, pesquisas vem sendo feitas para produtos além de cupulate, mas também farinhas provenientes do processo, contendo até 26\% de proteínas (Carvalho, 2004).

O principal motivo pelo qual as sementes do cupuaçu deveriam serer mais exploradas é devido os valores altos de proteínas $(9,80 \mathrm{~g} / 100 \mathrm{~g})$, lipídios $(63,13 \mathrm{~g} / 100 \mathrm{~g})$ e 
o seu valor de calorias totais $(671,73 \mathrm{kcal} / 100 \mathrm{~g})$. Porém um dos maiores problemas para extração do óleo de cupuaçu é devida a resistência da casca das sementes. Isso implica em uma comparação com o cacau que, apesar dos valores nutricionais semelhantes, ainda é negligenciado devido a densidade da casca da amêndoa do cupuaçu ser maior que a do cacau (Vilalba, 2003).

Conhecer e identificar essas técnicas pode ser o caminho para uma boa oportunidade de renda à agroindústria familiar relacionada a cultura do cupuaçu. Assim, o objetivo desta revisão é investigar através de referenciais bibliográficos os principais métodos de extração do óleo da semente de cupuaçu, com ênfase em metodologias acessíveis.

\section{ABORDAGEM}

Este trabalho foi elaborado por revisão bibliográfica de caráter descritivo sobre o óleo de cupuaçu e os diferentes métodos para extração do mesmo, com ênfase em metodologias acessíveis a agroindústria familiar rural. A coleta de dados foi realizada em setembro de 2018 nas bases de dados das plataformas Scielo, Google Acadêmico e a Base de Dados da Embrapa (Empresa Brasileira de Pesquisa Agropecuária) pesquisando, preferencialmente, artigos científicos. Foi detectado número reduzido de publicações sobre o assunto e, após seleção dos trabalhos mais relevantes, foram adotados os passos sugeridos por Souza et al. (2010) para esse tipo de revisão: leitura exploratória; escolha do material que se adequasse aos objetivos e ao tema deste estudo; leitura interpretativa e redação dos resultados.

\section{PRÉ TRATAMENTO DO FRUTO}

Os procedimentos envolvidos no processo de extração de óleo das sementes no geral se dividem em três etapas: pré-tratamento, extração e refino. O pré-tratamento consiste na limpeza das sementes, fermentação, secagem e moagem. A extração usualmente se faz por prensagem e/ou extração com solvente. $\mathrm{O}$ refino consiste em retirar resíduos deixando os óleos com um sabor ou cor mais agradável (Azevedo, 2001). 
Entre as etapas de pré-extração, propriamente dita, estão as etapas de limpeza das sementes que consiste na retirada de toda a polpa presente em torno das sementes, em seguida as sementes passam por fermentação normalmente em caixotes por sete dias com revolvimentos diários, após a fermentação as sementes são secas ao sol ou em forno para em seguidas passarem pela moagem que basicamente é a trituração dessas sementes para assim partir para o processo de extração. Assim as sementes estão prontas para a extração do óleo, que pode ser através de diferentes processos descritos a seguir.

\section{PROCESSO MECÂNICO DE EXTRAÇÃo DE ÓLEO DE SEMENTES DE CUPUAÇU}

O processo mecânico é feito através da compressão das sementes, feita por meio de um eixo girando de forma continua até a pressão máxima. Também pode ser feito por um cilindro hidráulico ou manual, através de parafusos ou de maneira elétrica. Vale ressaltar que os processos para extração de óleo das sementes de cupuaçu são normalmente com controle de temperatura, pois as sementes necessitam passar por extração a quente, o equipamento é encontrado no mercado por nome de prensa hidráulica com controle de temperatura (Pighinelli, 2010).

Algumas prensas mecânicas, já disponíveis no mercado, permitem controlar a pressão e a temperatura do sistema para maximizar a obtenção do óleo, bem como controle diferenciado para cada tipo de semente. Este método de extração mecânica não é utilizado exclusivamente para sementes de cupuaçu, mas para outras sementes, como por exemplo sementes de andiroba que são mais populares ao norte do país, mas como também é utilizada para sementes de uva. Algumas sementes necessitam de calor, com temperaturas próximas a $50^{\circ} \mathrm{C}$ e outras podem ser prensadas a frio (Pighinelli, 2010). 


\section{PROCESSO QUÍMICO DE EXTRAÇÃO DE ÓLEO DE SEMENTES DE CUPUAÇU}

Os processos químicos de extração são os que passam por tratamento com solventes desde a extração até o refino do material. Nas etapas de tratamento e pós tratamento são utilizados produtos como hexano e hidróxido de sódio. São raramente utilizados em agroindústrias ou como método caseiro de extração, porém podem ser efetuados em industrias de pequena escala.

Este método consiste preparação das sementes através da secagem em estufa até o peso constante, trituração e extração pelo método de arraste por solvente em um sistema de extração do tipo soxhlet. Em seguida a mistura é aquecida com MgO e H2O. Isso resulta em uma massa, também citada como bolo, que passa pelo processo de clarificação por adição de $\mathrm{Pb}(\mathrm{OAc})^{2}$ e o resultante é filtrado. Essa solução passa por um evaporador rotativo e é extraída com $\mathrm{CHCl}_{3}$. Por fim, após passar por processos de evaporação, o resultante gera a gordura de cupuaçu. Esse processo pode chegar até um rendimento de $59,2 \%$, ou seja, através do processo químico pode-se obter mais de $50 \%$ de gordura em relação ao peso total das sementes (Vasconcelos, 1975).

Esse é um dos processos mais utilizados pela indústria, porém não é o único processo químico existente para extração dos óleos vegetais. Outros ácidos também podem ser utilizados ou em alguns casos se alteram somente as concentrações.

\section{ASSOCIAÇÃO DOS MÉTODOS QUÍMICOS E MECÂNICOS PARA O PROCESSO DE EXTRAÇÃO DE ÓLEO DE SEMENTES DE CUPUAÇU}

Também chamado de extração supercrítica, consiste em elevar o solvente a condições altas de temperatura e pressão através de um sistema cilíndrico que trocam calor. Esse solvente é levado até o bolo de cupuaçu (soluto), onde ocorre a transferência de massa. Após essa mistura, ela passa por um separador que baixa a temperatura fazendo com que o soluto precipite. Essa técnica faz com que o solvente se misture mais ao soluto através do controle de densidade que é alterado com a mudança de pressão e temperatura (Azevedo, 2001). 
Dentre esses também se utiliza do método químico, em seguida passado na prensa para melhor aproveitamento de todo material presente na massa.

\section{MÉTODOS ADEQUADOS PARA A AGROINDÚSTRIA FAMILIAR}

Com o aumento da preocupação sobre assuntos como saúde e meio ambiente, é sempre importante priorizar técnicas que venham agregar valor a esses temas e que busquem não só o aproveitamento, mas que também tenham uma visão voltada também para o bem comum como sociedade em termos de saúde, sustentabilidade e preservação do meio ambiente. Assim, técnicas que agridem menos o meio ambiente e/ou que não causem prováveis malefícios a saúde do consumidor são sempre as preferidas para comercialização.

Entende-se a agroindústria familiar como uma atividade de produção de produtos agropecuários com conseqüente transformação destes em derivados alimentares de diversos tipos, ocorrendo, nesse processo, a agregação de valor ao produto final. Além disso, deve-se ressaltar que nestes empreendimentos há grande relevância do trabalho e da gestão por parte do próprio núcleo familiar que é o que empresta sentidos, significados e as estratégias que serão adotadas nesta atividade (Gazolla, Pelegrini, 2011).

Devido aos valores agregados ao princípio da agroindústria familiar, pode-se descartar o método puramente químico de extração, sendo um método bastante poluente, pouco sustentável, e que pode acarretar em malefícios a saúde. Já existem regulamentações a respeito do que é gerado como resíduos contaminantes no produto final das industrias, incluindo as indústrias de óleos.

Quanto a extração mecânica com associação ao químico, alguns trabalhos mostraram resultados no que se diz respeito ao processo de extração supercrítico ou mecânico associado ao químico. Devido ao fato de ocorrer um aproveitamento grande no processo de extração, conseguindo obter uma porcentagem alta de aproveitamento. Mesmo sendo um método que já vem sendo estudado desde os anos 80, alguns trabalhos citam o método de extração supercrítica como sendo um método de tecnologia limpa ao lado de métodos atuais existentes na atualidade (Meireles, 1997). 
Dentre as técnicas que foram citadas, a mais ecológica é a técnica de extração mecânica. Além de trazer o beneficiamento das sementes que são normalmente descartadas, o processo mecânico é limpo pois não se utiliza de solventes poluentes. Bem como gera um produto refinado ao ponto de ser útil para a alimentação humana.

[...] Para a extração da gordura de cupuaçu pede utilizar uma prensa hidráulica com capacidade para $60 \mathrm{kgf} / \mathrm{cm} 2$. As amostras de liquor de cupuaçu devem ser aquecidas em forno de microondas até a temperatura de $80^{\circ} \mathrm{C}$, acondicionadas em sacos de lona e colocadas dentro do cilindro da prensa. O tempo total de extração pode variar, mas leva em de 45 minutos, sendo que nos primeiros 5 minutos devese utilizar uma pressão menor de uns $10 \mathrm{kgf} / \mathrm{cm} 2$, em seguida aumentar para $20 \mathrm{kgf} / \mathrm{cm} 2$ por mais 5 minutos, $30 \mathrm{kgf} / \mathrm{cm} 2$ por mais 5 minutos e, finalmente, $40 \mathrm{kgf} / \mathrm{cm} 2$ no tempo restante (30 minutos). Tal procedimento é necessário para que os sacos de lona não sofram ruptura. Após a extração das gorduras, estas devem ser filtradas para a retirada de possíveis partículas sólidas provenientes das amostras de liquor (Grimaldi, 1978).

Vale ressaltar que apesar de ser uma técnica considerada verde, essa técnica gera desperdício de óleo, uma vez que a prensagem não é suficiente para extração total do óleo das sementes.

\section{CONSIDERAÇÕES FINAIS}

Visando a utilização da metodologia de extração de óleo de sementes de cupuaçu por agroindústrias familiares, acredita-se que a melhor técnica seria o método mecânico de extração.

\section{AGRADECIMENTOS}

Agradecemos ao mestrado acadêmico em Agroecologia (UERR, EMBRAPA e IFRR) pela oportunidade de ampliar o conhecimento em torno desse universo. E a CAPES pela bolsa de pós-graduação. Além de principalmente a EMBRAPA - RR pela oportunidade de estágio no laboratório de pós colheita. 


\section{REFERÊNCIAS}

ALVES, R. M.; FILGUEIRAS, G. C.; HOMMA, A. K. O. Aspectos socioeconômicos do cupuaçuzeiro na Amazônia: do extrativismo a domesticação. In: Mercado, cadeia produtiva e desenvolvimento rural na Amazônia. SANTANA, A. C. Belém: Edufra, 2014.

AZEVEDO, Alvaro Bandeira Antunes. Extração e fracionamento da gordura de cupuaçu das sementes com fluidos supercríticos I Álvaro Bandeira Antunes de Azevedo.-Campinas, SP: [s.n.], 2001.

CARVALHO, Ana Vânia. Extração, concentração e caracterização físico - química e funcional das proteínas de semente de cupuaçu (Theobroma grandiflorum Schum). Tese apresentada a Faculdade de Engenharia de Alimentos da Universidade Estadual de Campinas. - Campinas -SP, 2004.

DAL RI, Estela Sebastiany. Avaliação do processo produtivo e da qualidade de polpas de frutas comercializadas em Boa Vista/RR. 2006.

GAZOLLA, Marcio; PELEGRINI, Gelson. As experiências familiares de agroindústrialização: uma estratégia de produção de novidades e de valor agregado.

Ensaios FEE, Porto Alegre, v. 32, n. 2, p. 361-388, nov. 2011.

GRIMALDI, J. Les possibilités D'amélioration des techniques D,,ecabossage et de fermentation dans le processus artisanal de La préparation du cacao. Café, Cacao, Thé, v.22, p.306-316, 1978.

GONDIM, Tarcísio Marcos de Souza...[et al.]. Aspectos da produção de cupuaçu. Rio Branco - EMBRAPA - ACRE, 2001.

LANNES, Suzana Caetano da Silva; MEDEIROS, Magda Leite; AMARAL, Renata 
Formulação de "chocolate" de cupuaçu e reologia do produto líquido. Revista Brasileira de Ciências Farmacêuticas, Brazilian Journal of Pharmaceutical Sciences. vol. 38, n. 4, out./dez., 2002.

LIMA, H. E. de; CHAGAS, E. A.; SANTOS, V. A. dos; RODRIGUEZ, C. A.; ARAÚJO, M. da C. R. Severidade da vassoura de bruxa em genótipos de cupuaçuzeiros cultivados em sistema agroflorestal $(\mathrm{SAF}$ ?s) e produção de genótipos tolerantes à doença.

Repositório -- BDPA, 2013.

MAIA, G. A.; SOUSA, P. H. M. \& LIMA, A. S. L. Processamento de Frutas Tropicais.Fortaleza: Edições UFC, 320 p. 2007.

MEIRELES, M. Angela A. Extração Supercrítica de Óleos Essenciais de Condimento Usando Dióxido de Carbono. Boletim da SBCTA, vol. 31, no. 1, pp. 9- 14, 1997.

MEDEIROS, M. L. et al. Sorption isotherms of cocoa and cupuassu products. Journal of Food Engineering, v. 73, p. 402, 2006.

PIGHINELLI, Anna Leticia Montenegro Turtelli Estudo da extração mecânica e da transesterificação etílica de óleos vegetais / Anna Leticia Montenegro Turtelli Pighinelli. Campinas, SP: [s.n.], 2010.

RUSCHEINSKY, Nirse. Estudo da crsitalização e fracionamento térmico a seco da gordura de cupuaçu (Theobroma grandiflorum Schumann) I Nirse Ruscheinsky.Campinas, SP: [s.n.], 2005.

SANTOS, Neemias Dutra Dos. Análise comparativa da quantidade de lipídios da amêndoa do cupuaçu e da amêndoa do cacau. Neemias Dutra Dos Santos. -AriquemesRO, 2016. 
SAID, Maricleide Maia. Aspectos culturais e potencial de uso do cupuaçu (Theobroma grandiflorum (Willd. Ex Spreng. Schum.) no estado do Amazonas / Maricleide Maia Said.

- Manaus: UFAM, 2011.

VASCONCELOS, M. N. Lima et al. Estudo químico das sementes do Cupuaçu. Ac:fA AMAZONICA 5(3) : 293-295 . 1975.

VILALBA, Fábio de Albuquerque. Fragmentação mecânica de amêndoas de cupuaçu (Theobroma grandiflorum) por meio de um beneficiamento de cilindros / Fábio de Albuquerque Vilalba - Campipnas, SP. 2003. 